\title{
CHALLENGES OF TREATMENT-RESISTANT DEPRESSION
}

\author{
James Paul Pandarakalam \\ Department of Psychiatry, Northwest Boroughs Healthcare NHS Foundation Trust, Hollins park Hospital, \\ Warrington, United Kingdom
}

received: 16.7.2018;

revised: 10.8.2018;

accepted: 4.9 .2018

\begin{abstract}
SUMMARY
Guidelines for the management of treatment-resistant depression (TRD) do not meet the criteria of evidence-based medicine and better-quality research is required to inform clinical practice. Current treatments of resistant depression remains largely empirical. There are no bench-mark antidepressants. Clear and justifiable rationale should be followed while initiating new treatment strategies; systematic planning and careful monitoring of progress implemented while new treatment components are added. Biological psychiatrists should give due importance to the non-biological aspects of depression and psychotherapists should not overlook the biological correlates. Unidimensional solution will not work for a complex illness like refractory depression and a single answer should not be sought as a cure because the aetiology of depression is multifactorial and the pathophysiology itself remains unknown. Psychopharmacological interventions are still the main stay of treatment of TRD. There are two major alternatives to pharmacotherapy: neuromodulation and psychotherapy. Alternative terminologies for TRD like MTR-MDD (Multiple Therapy Resistant-Major Depressive Disorder) are being introduced reflecting the frustrations of clinicians and patients with the conventional definition of TRD and treatment modalities.
\end{abstract}

Key words: depression - antidepressants - psychotherapy - ECT - TMS - VNS - healing therapies

$$
* * * * *
$$

\section{INTRODUCTION}

Depression is a highly controversial topic in psychiatry as there are long-standing disputes between the biologically and the analytically oriented psychiatrists. At present it is not advisable to hold any strong views about this universal phenomenon with its varied transcultural aspects. Depression may be a psycho-bio, social condition, but there may be contribution from non-ordinary states of consciousness to their aetiology (Pandarakalam 2018). The popular misconception that depression is due to lack of will power has contributed to the stigma of this affliction (Bhugra 2013). Depressive illness has become time-consuming and a financial burden for all the health systems. TRD patients have been reported to have significantly higher medical costs and to be twice likely to be hospitalised resulting in a 6fold increase in overall medical costs compared to nonTRD patients (Crown et al. 2002). In the year 2000, the total cost of adult depression was estimated at over $£ 9$ billion in England and the direct treatment costs were $£ 370$ million (Thomas \& Morris 2003). In fact, depression including the treatment of co-morbid conditions are estimated to have cost the U.S. economy more than \$210 billion in 2010 (Greenberg et al. 2015).

Depression and suicide are connected, with an estimate that up to 60 percent of people who commit suicide have major depression. Clinical depression is associated with social, occupational and physical impairment and mortality, despite the recent advancements made in its treatment. Older studies using stricter definitions showed the incidence of suicides linked to major depression was around 15 percent. Unipolar depression and hopelessness is among the most frequently apprehended risk factors for suicidal thoughts and behaviours (Ribeiro et al. 2018).

\section{DEFINING AND ASSESING TREATMENT RESISTANCE}

A major depressive disorder (MDD) is indicated by the presence of at least five of the following symptoms (American psychiatric association 2013) occurring independent of physical illness, normal bereavement, alcohol or drugs:

- Abnormal depressed mood;

- Abnormal loss of interest and pleasure;

- Appetite or weight disturbance;

- Sleep disturbance;

- Disturbance in activity (agitation or slowing);

- Abnormal fatigue or loss of energy;

- Abnormal self-reproach or inappropriate guilt;

- Poor concentration or indecisiveness;

- Morbid thoughts of death or suicide.

At least one symptom must be abnormal depressed mood or loss of interest and pleasure, persisting for most of nearly every day for at least two weeks and significantly impairing function and daily life. When fewer than five symptoms are present, the disorder is called minor depression. Dysthymia is defined by the presence of at least three symptoms, including depressed mood, on most days for at least 2 years. 
Table 1. Assessment of treatment- refractory depression Re-evaluate current treatment

Adequate trial given? Suboptimal dose and non-adherence lead to pseudo resistance

Exceed BNF limits (in specialist centres only)

Check blood levels if facility available

Review the differential diagnosis- sub-clinical bipolar disorder?

Assess for psychotic symptoms

Exclude schizoaffective disorder

and non-affective disorders

Partial response or no response

Re-evaluate personal history

Explore interpersonal and family dynamics

Exclude soft bipolar disorder

Investigate co- morbid physical conditions (hypothyroidism, cushing's syndrome, parkinsonism, malignancy, anaemia, viral infections, vitamin deficiencies, and dietary deficiencies etc)

Co-morbid psychiatric conditions:

- Substance misuse, dependency-alcohol can cause tryptophan depletion;

- Anxiety disorders;

- Eating disorders;

- Personality disorders;

- Post traumatic disorders.

Assess suicidal risk

Asses pre-morbid personality

Concurrent non-psychiatric drug usage

eg. Methyl dopa, beta blockers, reserpine, steroids, immunosuppressants, anticholinergics, sedatives etc.

Organic factors

Explore the maintenance factors

Investigate compliance

Check the negative pressure from the part of the relatives and friends reg. medication intake.

Variable day routines and travelling habits affect dosing

Examine whether the team's expectations are realistic, eg time scale for improvement.

Asses motivation of the patient - it is the

very nature of the illness to be hopeless

There is not a standard definition for treatment resistance. It may be defined as an unsatisfactory response to two adequate trials of two different classes of antidepressants at optimum dosage for sufficient duration (Thase \& Rush 1997, Souery 1999). Some consider treatment resistance only after trials of several different classes of antidepressants (Judd 2000) or even electroconvulsive therapy (Fink 2001). But there are no strict criteria to measure clinically meaningful improvements and the number and type of treatment trials that a patient should experience before being labelled as refractory depression. Multiple factors are involved in treatment resistance (Table 1). Several severity indicators, such as longer duration of depressive episode, moderate-high suicide risk, anxious comorbidity, high number of hos- pitalizations, age factors and higher dosage of antidepressants may account for non-response to multiple therapeutic interventions (Vera et al. 2016).

Professor Anderson has opined that the standard definition of TRD raises several questions (Anderson 2018). He questions the rationale of specifying two antidepressants and the meaning of an adequate trial. He also wonders whether sequential drugs from the same and different antidepressant classes be treated as the same. Professor Anderson is also seeking the validation of how to incorporate psychological therapies and alternative medicines in the current treatment regime of TRD. He thinks that treatment adherence, intolerance, partial response and non-response in past episodes of depression also matters but not given specifications in the criteria for TRD (Anderson 2018). Psychological resistance is sometimes not distinguished from biological resistance. Over the last few decades, the assessment of TRD has improved, but because of lack of consensus, clinical and nosological debate continues (Fornaro et al. 2010).

\section{NEWER TERMINOLOGIES}

Despite newer medications, TRD literature indicates that the investigatory endeavours came to a standstill a decade ago. TRD field has failed to progress beyond classifications and treatment strategies (Moller et al. 2013). A new vocabulary is needed to define this terrible and disabling condition. Moller et al. suggest borrowing prefixes from general medicine and name treatment resistant nonpsychotic major depression as malignant, pernicious or virulent nonpsychotic depression and psychotic TRD could be termed malignant, pernicious or virulent psychotic depression (Moller et al. 2013).

McCallister and colleagues propose the development and adoption of another useful terminology as one step toward improving medical treatment. They suggest another terminology- MTR-MDD (Multiple Therapy Resistant Major Depressive Disorder). These recent proposals to modify the very time old terminology TRD is welcoming. The newer terms could spawn productive discussions and re-evaluations of medical practices. These labels could get further modified as research progress. Depression needs urgent timely intervention because of its spiralling nature in the psychological and social sphere; cognitive distortions spiral down with their domino effect and social causes lead to loss of job and breakdown of relationships to the detriment of the sufferers.

\section{RESPONSE TO PHARMACOTHERAPY}

Between 20 to $40 \%$ respond only minimally to monotherapy (Shelton 1999). Only 50\% of patients not responding to a primary antidepressant improve when another antidepressant is introduced (Depression Guideline Panel 1993). Faster onset of action and greater therapeutic efficacies with minimal side effects are the main prescription- criteria of antidepressants (Table 2). 
Table 2. Key points of selection of antidepressants

\author{
Fast onset of action \\ Sustained response \\ Sustained remission \\ Sustained prevention of relapse \\ Monotherapy \\ Single regime \\ Better tolerability \\ Fewer discontinuation symptoms
}

Early detection and vigorous treatment with aggressive treatment of residual symptoms followed by continued maintenance treatment should be the policy of managing pharmacotherapy resistant depression (Trivedi \& Kleiber 2001).

To avoid a higher chance of adverse reactions; clinicians tend to prescribe a low dose of antidepressants even though such a practice reduces the chance of improvement slightly, but this could lead to partial response. It is important to distinguish partial response and no response for the future course of treatment. Past drug response, adverse effect profile differences, concomitant medical disorders and concurrent drug therapy are some of the considerations to be well-thought-out while choosing between switching and combination/ augmentation therapies. Switch is indicated for no response and those experiencing adverse effects; partial response signal combination therapy or augmentation. However, switching is less effective than augmentation (Posternack \& Zimmerman 2001).

Comorbidities such as substance abuse, personality disorders, and general medical conditions including hypothyroidism, anaemia etc can also greatly influence the overall success of a treatment plan. Genetic and metabolic variations that may make the biology of the patient unique contributing to poor response to medications should also be considered (Perils 2008). Despite the fact that phenomenal advances have been made in genetic testing, these tools have not been established for guiding psychiatric care. Multiple-loci genotyping tests are anticipated to provide information regarding patients at higher risk of TRD, drug related side effects and the recurrent nature of MDD (Serretti et al. 2011).

\section{INCIPIENT BIPOARITY}

Unrecognised or bipolarity in disguise is another issue to be excluded when challenging TRD and when bipolarity is diagnosed, addition of mood stabiliser is the first step towards treatment or even therapeutic diagnosis. Bipolarity should be screened in all cases of monopolar depression (Table 3). While this cannot be always accurate, one way of preventing treatment emergent bipolarity is co prescribing antipsychotics or mood stabilisers to prevent hypomanic switch. Such a view needs further investigation. Some antipsychotics and mood stabilisers have more anti manic properties than others.
Table 3. Features of incipient bipolarity

Non-response to antidepressants

Family history of bipolar disorder

Family history of completed suicide

Psychotic features

Atypical symptomatology

Retarded presentation

Higher suicidal risk

Violent mood swings

Co-morbid anxieties

Past history of mood elevation

Thinking people are unfriendly

Legal problems

An adjunctive therapy has the advantage of negating the treatment emergent agitation. Without sufficient knowledge, experience and care, doctors can deteriorate as licensed drug dealers and a sound knowledge of psychopharmacology is essential for general practitioners in these days of shift of psychiatry from the secondary care to primary care. The risk of suicide is higher among bipolar patients than monopolar patients and this is particularly so in the mixed affective states. The newer antidepressants unmask incipient bipolarity and most often result in mixed state. This may be true of younger adults while the older depressed adults would have more likely declared their mania.

\section{DIFFERENT TREATMENT STRATEGIES}

Now that there are a variety of medication and treatment strategies, the good news is that most patients have the potential to respond to treatment (Table 4). Full symptom remission (wellness) and return of optimal psychosocial function with minimal side effect burden is the clinical goal of treatment of prophylaxis resistant depression. Increasing the dose of the antidepressant carries the unavoidable risk of increasing the chances of adverse effects but can also have favourable effects. In the same vein, it has to be stated that the clinician may be thrown into the dilemma that increasing the dose may delay the recognition of early state TRD and increase the incidence of discontinuation symptoms in the event of discontinuing it due to failure of response (Kasper \& Montgomery 2013). The evidence supporting the benefits of different treatment strategies is oddly slender and clinicians should have realistic expectations and tolerance to deal with ambivalences.

\section{DRUG SUBSTITUTION}

A change from one antidepressant to another one in the same class seldom produces any additional benefit whereas switching to an antidepressant with a different mechanism of action has proven to produce more impressive response rate. Switching from a tricyclic to 
an alternate antidepressant agent has been always shown better efficacy. Because SSRIs are structurally diverse, switching from one SSRI to another may also be logical. SNRI is worth considering if an SSRI fails. If the halflife of the first SSRI is quite long, switching without a prudent wash out period can cause drug interactions.

When switching antidepressant drugs, one of the problems is discontinuation symptoms and disappointment from the part of the patients that they have lost some initial benefits gained out of the first drug in addition to the disadvantage that patient must cope with another waiting period for the substituted drug to produce desirable results. Switching is found effective if the clinical syndrome is atypical. In the past, M.A.O. inhibitors and I.V. Clomipramine were popular treatment for TRD.

The term combination and augmentation are used interchangeably. In general, combination refers to the use of more than one type of disease specific treatment applied to the therapeutic management of an illness (Shelton 2002). Thus, combination therapy involves the addition of a second antidepressant to the therapy regime and is different from adjunctive therapy in the sense it means to employ a second agent to reverse an emergent side effect or obtain a complimentary clinical effect. Augmentation involves the use of a non-antidepressant agent along with the antidepressant. The modus operandi of this approach (combination and augmentation) is that two different treatments together may have different mechanism of action and therapeutic response which is different from either drug alone.

Table 4. Strategies for managing refractory depression Optimise dose, if partial response

Lengthening therapy

Use what has worked for the patient in the past.

Drug substitution

- SSRIs. These are agents of first choice due to ease of use, more tolerable side effects and safety in overdose;

- Another class-venlafaxine, mirtazapine;

- High dose tricyclics.

Combination of classes

- SSRI + reboxetine;

- SSRI + tricyclics.

Augmentation

Electro convulsive therapy

Retrial of ECT for those previously administered ECT

Trans- cranial magnetic therapy

Vagal nerve stimulation

Psychotherapy

Light therapy and medication

Physical activities and medication

Sleep deprivation

Consciousness based healing

Neurosurgery is still available for severe

treatment-resistant depression.

\section{COMBINATION}

The use of combination therapies is on the increase because of more confidence and experience with antidepressants. Dual antidepressant treatment strategy has the disadvantage of drug interactions. Even newer generation antidepressants have only $70 \%$ efficacy rate, a figure which includes partial and full response. A significant number of depressed patients who do not adequately respond to SSRIs could benefit from the addition of their therapeutic predecessors-tricyclic antidepressants, but they are all small studies. Noradrenergic one is the choice for combination with the SSRI.

It is now increasingly recognised that SSRIs are not as effective as tricyclic antidepressant therapy in certain subsets of depressed patients, indicating the importance of norepinephrine re uptake inhibitors in the management of such patients. The recent explosion of SSRIs camouflaged the role of nor adrenaline in the causation of depression. Noradrenaline may preferentially improve vigilance, motivation and self- perception. There is evidence from controlled investigations that venlafaxine, an SNRI. is effective in treating treatment resistant depression. One way of achieving the clinical effect of an S.N.R.I is by combining an SSRI with an NRI, thereby converting a narrow spectrum antidepressant to a broad spectrum one (Devarajan \& Dursun 2000, Dursum \& Devarajan 2001). SSRIs and SNRIs may be working by parallel and independent pathways even though there are suggestions without clear evidences that NRIs influence depression by indirectly facilitating serotonergic transmission and SSRIs act by facilitating noradrenaline.

Tricyclic antidepressant therapy is associated with relative risk but reboxetine has a good safety profile. The safer side effect profile of reboxetine bodes well for long term patient compliance. Citalopram is a chiral antidepressant and is found to be effective in combination with Reboxetine (Forbes \& Rogers 2003). Its eutomer, escitalopram appears to be suitable for combination therapy. In the olfactory bulbectomized rat model of depression, reboxetine, sertraline and their combination were tested and, the combination treatments had better outcome (Harkin et al. 1999). Reboxetine is not cardiotoxic and it is not associated with an increased risk of seizures or orthostatic hypotension. Reboxetine has mild anticholinergic effects and produces sexual side effects. It is safer in overdose and seems to have negligible interference with the pharmacokinetics of other drugs. Venlafaxine and mirtazapine combination has been dubbed as 'California rocket fuel' and DuloxetineMirtazapine combination as 'Limerick rocket fuel.'

\section{AUGMENTATION}

Patients with depression respond variably and unpredictably to various antidepressants. There are now a plethora of clinical trials showing the efficacy of nonantidepressant agents. Augmentation therapy is an active 
Table 5. Combination/augmentation

Patients unresponsive to the initial antidepressant may achieve clinical response when the second agent is added.

Discontinuation symptoms due to withdrawal of the original antidepressant avoided and patient does not have to cope with another waiting period for the substituted drug to produce desirable results.

The strategy builds on therapeutic gains obtained with the primary antidepressant and allows patients to continue to reap whatever benefits they have from the original drugs but with the additive or synergetic benefits of the augmentor or the additional combined antidepressant; switching has the disadvantage of losing the little gain already obtained.

Switching requires care in the changeover of drugs, which can cause delay and discontinuation reactions; these are avoided with the addition of a second drug.

Second compound is generally well tolerated and does not substantially alter the side effect profile of the first antidepressant.

Rapid onset of antidepressant action

Response rate is comparable or superior to substitution which involves tapering off the first drug wash out and delay in onset of the second drug.

Disadvantage is reduced concordance and increased side effect as a result of taking two agents than one.

area of research and has certain advantages over switching (Table 5). Different classes of drugs are taken advantage for augmentation.

\section{Lithium}

Lithium is essentially used for preventing recurrence of mania and depressive disorder. Lithium has several pharmacological actions and it is unclear which of these explains the therapeutic effect. Its effect in increasing brain 5HT has clinical relevance. Lithium is thought to enhance serotonin transmission by the activity of postsynaptic serotonin or 5HT receptors. This reduces the negative feedback of serotonin-releasing cells and thereby increases serotonin levels in synaptic cleft. Lithium may also have favourable effect on other neurotransmitters and neuromodulators (Montigny 1994). The therapeutic dose and toxic dose of lithium are close together and that means regular scrutiny of its plasma measurement during treatment. Because of the side effects of lithium, this treatment strategy is underutilized. Lithium augmentation to an ongoing antidepressant trail was widely used in the 1980s and early 1990s. Low dose (300mg to $600 \mathrm{mg}$ per day) to higher doses were used to treat antidepressant resistant depression. But there has been a disenchantment with its use recently as clinicians found only acceleration than augmentation response (Fredman et al. 2000).

Lithium augmentation has been the most widely studied strategy in the literature of treatment involving
11 published double-blind trials and most of the studies involved tricyclics, but the clinical predictors of its efficacy regarding polarity and bipolarity are poorly understood. To this effect, a randomised controlled study revealed that lithium augmentation was more effective in patients with a final diagnosis of bipolar disorder than with MDD and subjects with more than three major depressive episodes showed a significant response to lithium augmentation (Sugawara et al. 2010). More recently, randomized controlled trials with more than 30 open-label and comparator studies have confirmed the efficacy of this combination and the rapidity of response (Bauer 2010).

\section{Anticonvulsants}

There is an observed shift of prescription practice from lithium to valproate across the Atlantic and is not based on reliable evidence of efficacy but, it is justifiable because of the safer side effect profile of valproate. Augmenting antidepressants with anticonvulsants has shown variable effects in trials. Carbamazepine has only a weak antidepressant property. There is an increasing interest in glutamate action although these drugs are essentially anticonvulsants.

Lamotrigine sold as the brand name Lamictal is a glutamate enhancing drug and is a potentiating agent for antidepressants. It is associated with more antidepressant potency than either carbamazepine or valproate. Carbamazepine induces enzymes that facilitate the metabolism of lamotrigine; blood levels of lamotrigine are somewhat lower when these two agents are taken together. Valproate could double plasma levels of lamotrigine. It has usefulness in treating bipolar depression and TRD. Among the well tested anticonvulsant in augmenting antidepressants, lamotrigine stands first (Barbee et al. 2011, Ivkovic et al. 2009). Isolated cases where lamotrigine has been more effective in treating TRD than ECT are reported (Mihara et al. 2016).

\section{Antipsychotics}

Typical antipsychotic used in conjunction with antidepressants have been found to have only modest effect in the treatment of depression. Clinical trial employing augmenting SSRIs with atypical antipsychotics hint that this novel augmentation strategy is a promising therapeutic avenue. Risperidone (Ostroff \& Nelson 1999), olanzapine (Shelton et al. 2002) and most other atypical antipsychotics are now used by many clinicians to augment the effect of SSRIs. In Europe, quetiapine has been approved as an add-on medication to the antidepressant treatment and aripiprazole or olanzapine along with fluoxetine have been used in USA for MDD (Kasper \& Montogomery 2013). Neither the use of SSRI nor the novel antipsychotic alone produces the effect similar to that produced by the combination of the two.

The biological or chemical mechanism for the beneficiary effect of these combinations are not clear. Novel antipsychotics are potent serotonin-2A receptor anta- 
gonists, which is similar to the effects of some of antidepressants. Another hypothesis is based on the data suggesting that serotonin binding to $2 \mathrm{C}$ receptors inhibits the release of dopamine and norepinephrine in the frontal cortex preventing serotonin from binding to 2 $\mathrm{C}$ receptor sites that may result in increased dopamine levels in the prefrontal cortex and nucleus accumbens. Novel antipsychotics are also serotonin -2 Cblocking. The combination of antipsychotics and SSRIs antagonise serotonin receptors and elevate the frontal cortex dopamine levels. These effects could probably explain the additive effects on depression.

\section{Thyroxine}

There are numerous clinical reports favouring the view that thyroid hormones can influence mood and changes of thyroid status has bearing to depressive symptomatology; the relation between hypothyroidism and depression is well established (Bauer et al. 2008). Thyroid hormones may modulate the effect of antidepressants. Both T3(triidothyroinine ) and T4 (levothyroxine) are used for augmenting the effect of traditional antidepressants. Efficacy of augmenting TCAs with triidothyroxine has been supportive in the early open trials. Studies of thyroid augmentation of SSRIs are not many and warrants further research. L-triiodothyronine is said to enhance sensitivity to noradrenergic receptors and is more active than $\mathrm{T} 4$. Depressed patients receiving T4 replacement sometimes respond only when $\mathrm{T} 3$ is added to their treatment regimen (Coper-Kazaz et al. 2007) but, the results have been inconsistent.

\section{Buspirone}

Buspirone when used in conjunction with an antidepressant is found to have antidepressant property (Jacobsen 1991). Buspirone acts as a full agonist at the presynaptic autoreceptor and as a partial agonist at the postsynaptic autoreceptor (Sussman 1995). Administration of buspirone decreases extracellular serotonin concentration over the short term through activation of 5HT1A presynaptic autoreceptors. Buspirone also activate postsynaptic 5-HT1A receptors. Regular buspirone administration desensitises and downregulate 5 HT1A presynaptic autoreceptors, but not postsynaptic 5-HT1A receptors thereby encouraging further serotonin release. Using serotonergic agents to augment SSRIs can result in serotonin syndrome (Fava 2001).

\section{Pindolol}

One of the mechanisms responsible for the delayed onset or lack of response to antidepressants is thought to be due to an initial increase in the 5HT concentration in the synaptic cleft prompting a negative feedback mechanism that decreases further release of the neurotransmitter; a process appearing to be mediated by presy- naptic somatoderitic 5HT $1 \mathrm{~A}$ receptors and this is the basis of strategies making use of 5HT1A antagonists. The betablocker pindolol which is a 5HT 1A-receptor antagonist has been used to augment antidepressant therapy; several open and controlled studies have yielded only mixed results warranting further investigation. Pindolol speed up the onset of SSRIs rather than augmenting the antidepressant effect (Maes et al. 1999).

"Triple therapy" (L-tryptophan+ lithium+ Antidepressant) of the $80 \mathrm{~s}$ is no more used. Psychostimulants such as amphetamine or methyldopa has only academic interest as antidepressants and not recommended in TRD. Likewise, L-dopa though helpful in promoting a degree of psychomotor activation has no proven antidepressant effectiveness. Adding clonazepam to SSRIs is found to reduce the lag time for the onset of antidepressant action. Positive results with hormonal/ omega-3fatty acid augmentation need to be replicated (Anderson 2003).

Transdermal Selegiline is thought to be effective for patients with atypical depression (Pae et al. 2007). Modafinil is included in the BAP guidelines for TRD for use in specialist centres (Cleare et al. 2015), but not included in the NICE. Pramipexole is another drug that may be used in TRD when other means fail and has endorsement in two conflicting RCTs (Kleebiatt et al. 2017). Intravenous Ketamine is another addition to the psycho-pharmacological armamentarium of TRD treatment (Bobo et al. 2016) but involves invasive procedure with limited duration of effect.

\section{BRAIN STIMULATION THERAPIES}

ECT is still considered as a gold standard for relieving depression by many clinicians who also believe that by not considering it sooner, psychiatrists are consigning many patients to less effective treatments and the risk of chronic illness. Much has been written about ECT. National institute guide lines (NICE 2003) states ECT should only be given to achieve a rapid, short-term improvement when other treatment strategies have failed, or when the patient's condition is potentially life threatening. One of the side effects highlighted is a temporary memory impairment-retrograde and anterograde amnesia. As this type of cognitive disturbance is encountered in the psychiatric condition itself, it is difficult to differentiate the effect of the ECT from the associated mental health problem itself.

Prolonging Remission in Depression in Elderly (PRIDE) study revealed that ECT combined with an antidepressant was effective in preventing relapses in patients 60 years and older who had severe depression (Kellner et al. 2016a, 2016b). ECT survived despite severe periodic attack from outside and inside the medical profession. ECT is a treatment which falls within the provisions of Section 58 (in England and Wales). It can be administered only if the patient consents or, if not, a second opinion must be sought. It can be administered under common law in an emergency. 


\section{TRANSCRANIAL MAGNETIC STIMULATION}

Like ECT, Transcranial magnetic stimulation (TMS) and Vagal nerve stimulation (VNS) employ electromagnetic energy to alter brain activity and are potential therapies requiring more study. They are not going to replace any other therapies but are another option. The research studies about TMS has risen exponentially whereas evidences for the effectiveness of VNS is still fragmentary. TMS is based on the use of pulsed magnetic field. Earlier TMS machines produced a pulse every three seconds and was developed as a diagnostic aid for neurologists. Modern machines produce up to 50 stimuli per second (rapid rate TMS or rTMS) and can modify the activity of specific brain areas; pathological neural activity could also be corrected.

TMS stimulation modulates the activity of cortical neurones. The excitability of the cortical areas can be increased or decreased by using the TMS stimulation and, combining TMS and neuroimaging techniques have demonstrated that functionally related sub cortical structures can also be influenced. It is assumed that left prefrontal cortex becomes less active in clinical depression and these areas are accessible to TMS stimulation. Preliminary reports suggest that TMS may be effective as an add on therapy similar to lithium augmentation or an alternative when pharmacotherapy fails (Reid et al. 1998). Unlike ECT it does not produce any cognitive impairment, does not require a general anaesthetic and not invasive. In 2008, FDA approved rTMS for use in moderate TRD (O’Reardon et al. 2007).

\section{VAGAL NERVE STIMULATION}

The use of anticonvulsants medications as mood stabilizers suggests a shared aetiology in convulsive and mood disorders. Vagus nerve stimulation (VNS) had its origin in serendipitous observation; VNS treated drug resistant epilepsy patients showed improvement in mood and cognition (Rosenbaum \& Henninger 2000). Vagus nerve is one of the information-highways to and from the brain. Central afferent projection goes to the nucleus tractus solitarius from where there are homological connections to several key structures that regulate affect (locus coerulus, thalamus, hypothalamus, amygdala and hippocampus). A generator about the size of a cardiac pacemaker is implanted in the patient's upper chest and connected via electrode to the left vagus nerve in the neck near the carotid artery. This device is programmed to stimulate every 3 to 5 minutes and the impulse can last for 30 seconds. The programmer can also be used to modify the electrical impulses in frequency, intensity, duration or to turn the impulses off altogether.

Adverse effects are minimal. Relationship of autonomic signals to limbic and cortical function has been of great investigatory interest to physically alter brain function and it is also hypothesized that VNS instigate changes in norepinephrine and serotonin, dopamine and GABA and normalization of $\mathrm{CRH}$-induced ACTH secretion. It may promote neural network activation or some sort of reinduction of synchronicity in brain waves or timing of various neural circuits. It is found to increase activity in the thalamus and brain stem. We must confirm whether it truly works before figuring out how it works. VNS is already used for treatment resistant patients with epilepsy in U.K. A European study claimed a response rate of $53 \%$ after 2 years (Bajbouj et al. 2010) and thus, not an acute treatment option for TRD.

\section{PSYCHOSURGERY}

Specialized centres for neurosurgical interventions are still available for chronic, severe and disabling depression that are completely refractory to all conventional therapies even though social, political and moral issues as well as certain scientific and philosophical questions of psychosurgery remain unanswered. Fears that psychosurgery was being used on minority and disadvantaged population for social control are unsubstantiated. The two main procedures used are anterior cingulotomy and subcaudate tractotomy and are aimed at severing the neural connections between the prefrontal cortex and the emotion centres of the limbic system. The theoretical basis for this treatment is not well established. Psychosurgery cannot be given to any patient, whether formal or informal, without the patient's consent and a second opinion.

\section{PSYCHOTHERAPY}

Even though antidepressants are the first line treatment for depression, only one third of patients respond fully to pharmacotherapy. An integral package of treatment of refractory depression should address the physical, psychological and social aspects of care. Interpersonal psychotherapy is to identify and change problems in social and personal relationship that contribute to depression. Psychodynamic therapy focusses on the past experiences and how they might be contributing to current mental state in ways of which the person is not conscious. Psychoanalysts have been trying to bring out past traumatic memories to find the cause of depression. Memories repeated get multiplied and become more emphatic. Patients would benefit by opening the "jewel box of the past" and not the "worm box".

\section{COGNITIVE THERAPY}

The effective non-pharmacological therapies are now lumped together as Psychosocial-interventions, they also include cognitive-behavioural approaches (Pandarakalam 
2004). Depressive cognitions are generally divided into three groups: 1) negative automatic thought, 2) a set of unrealistic expectations, 3) a series of cognitive distortions. Therapy resistant depressed patients who are trapped in the cob-web of cognitive distortions experience all emotions as sorrow like patients suffering from thalamic syndrome feel all their sensory experiences as pain.

The CoBalT randomised controlled trial demonstrated that among patients who have not responded to antidepressants, augmenting usual care with CBT is effective in reducing depressive symptoms, and these effects, including outcomes reflecting remission, are maintained over 12 months (Wiles et al. 2014). The intervention was cost-effective based on the National Institute for Health and Care Excellence threshold and they also noted that patients may experience CBT as difficult but effective. An economic evaluation of the CoBalT trial revealed that adding CBT to pharmacotherapy for patients with TRD could substantially alleviate the burden of the disease (Hollinghurst et al. 2014).

After analysing the literature pertaining to the effectiveness of various forms of psychotherapy in patients who have not responded to antidepressant pharmacotherapy, Kasper and Montgomery reveal that patients who have not responded to one or two trials of antidepressant medication have a 30-50\% chance of responding to a focused psychotherapy (Kasper \& Montgomery 2013) recognizing that diagnosis of treatment-resistant depression far outstrips the development and availability of effective treatments. Well-controlled and adequately powered studies have not yielded satisfactory evidence to support focussed psychotherapy and this is a hindrance in integrating such modalities into evidencebased algorithms for TRD (Trivedi et al. 2009, Gaynes et al. 2011). Psychodynamic psychotherapy for TRD has also started re-emerging (Driessen et al.2010) and possibly indicates the frustrations of managing this potentially malignant condition.

\section{ALTERNATIVE MEDICINES}

The current popularity of alternative medicines and complementary therapies reflect the despair and helplessness of patients with severe refractory depression (The term "Complementary medicines" is sometimes used to comprise both alternative medicines and complementary therapies). Herbal medicine with its branches of Bach's flower remedies, Chinese and Ayurvedic medicine, Homeopathy and Unani are a few of the alternative medicines available in U.K.

St John's wort (Hypericum perforatum) has gained great media attention. It contains a combination of at least ten different components including hypercins, flavonoids and xanthons. Investigators conclude that it has no usefulness in severe depression (Davidson et al. 2002). Some studies have been unable to differentiate hypericum from placebo (Montegomery 2000, Shelton
2001). There is a risk that people with clinically significant depression may self-medicate with hypericum rather than receive medication. Concerns have been raised about adverse interactions of hypericum with certain drugs.

Readily available herbal medicines have led to confusing choices for patients. Valeriana and Kava Kava are two other herbal remedies for depression without any trial data. Other alternative medicines have also no proven value in "difficult to treat depression". Many people are not aware that such remedies can cause side effects or interact with other drugs. Alternative medicines are more popular among the inner-city population and among the ethnic minorities.

\section{COMPLEMENTARY THERAPIES}

Parallel to the marketing of newer antidepressants, complementary therapies have also, been flourishing and they are very popular among patients suffering from resistant depression. Most of them are based on the principles of progressive muscular and mental relaxation. If anxiety is the first symptom to manifest in depression, it is also the last symptom to disappear and practioners of complementary medicines are probably helping to relieve the anxiety component of this mental illness but some of them exploit such patients offering false expectations. Hypnotherapy, Reflexology, Aromatherapy, Reiki, Osteopathy, Acupuncture are some of the complementary therapies practiced in U.K. and interestingly they are popular even among the highly educated.

Among the complementary therapy techniques, hypnotherapy seems to have gained a renaissance in recent years. Hypnosis does not relieve the psychic pain of depression; it has no value in correcting biological symptoms. One of the meta-analytical studies has established that hypnosis enhances the efficacy of both psychodynamic and cognitive behavioural therapies (Kirsch \& Lyme 1995). But hypnosis is not a panacea or a substitute for physical and various psychotherapeutic treatment methods. Kirsch (1996) considers hypnotherapy an empirically validated, nondeceptive placebo and the effects are mediated by response expectancies. This is probably one of the several psychological mechanisms that explain the working of hypnosis. Hypnosis is contra-indicated in actively suicidal patients and patients with psychotic features. The traditional view in using hypnotherapy for depressed patients are that of extreme caution and not favourable to its unitary use.

Some evidence exists to think broad spectrum light exposure used for the treatment of seasonal affective disorder has efficacy in non-seasonal depression or as an augmenting agent with antidepressant medications. Physical activity and exercises also augment other antidepressant treatment methods. Medications resistant depressions ultimately evolve as a form of dysthymic 
disorder with multiple physiological and psychological symptoms and, tend to be episodic, recurrent and have a longitudinal course. Whether treated or untreated, chronicity is ascribed to depression when it runs beyond two years.

\section{HEALING DEPRESSION}

With the progress in consciousness studies, the view that depression is a disorder of consciousness is getting popular and consciousness-based medicine has started emerging in different quarters of healing professions. Spiritual therapies are also gaining ground as a last resort for therapy resistant depression. Change of life style and a philosophical outlook towards life are the basic principles of such therapies. Depression must be neutralised with medications and positive life events but has to be rehabilitated at a deeper level where medications may not reach, and spiritual therapies become relevant in such inner healing.

The U.S. anaesthetist, Dr Rajiv Parti who had a near death experience is a strong advocate of such a healing medicine and claims that consciousness-based medicine he promotes is based on several widely held perpetual truths (Parti 2016). They may include the belief that consciousness exists outside of the body, there is life after death, we are all connected to each other and celestial beings exist to help and guide us. In downward causation of depression proposed by non-reductionists as opposed to the upward causation of depression promulgated by reductionist scientists, the biological correlates of depression become an epiphenomenon of an underlying deranged psycho-spiritual activity. Their emerging school of thoughts would involve spiritual resistance along with the psychological and biological resistance as a feature of TRD. The subjective experience of depression may respond to consciousnessbased healing methods, but the tormenting psychic pain must be addressed through the highly precious psychotropic medications. But, some healers believe that pharmacotherapy might silence the spirit and therein lies the perils from poorly trained spiritual therapists.

In the past, insightful patients used to claim that there is something wrong with their mind, but now days, patients state that there is something dysfunctional about their brain and want a quick fix. Such patients require several months of preparation to become psychologically minded to receiving some form of psychological therapies whereas they might require years of preparation to become spiritually minded for spiritual therapies.

\section{FUTURE DIRECTIONS IN PSYCHOPHARMACOLOGY}

New techniques of fluorescence histochemistry- immunohistochemistry and in situ hybridization has permitted the elucidation of chemically defined neural circuits providing tools for burgeoning field of neurochemical pathology. Consequently, novel biological concepts of depression have germinated recently, and they involve other receptor systems or intracellular targets. Branching out of the current monoamine cyclebased antidepressants, the possibility of non-monoaminergic antidepressants is being explored. During recent years many new potentially relevant brain transmitters and proteins have been identified.

There are several neuropeptide-based approaches to develop novel antidepressants (Iversen 2003). They are substance $\mathrm{P}$ antagonists, vasopressin antagonists, melanocortin-concentrating hormone antagonists and corticotrophin-releasing factor receptor antagonists. Various recent findings show that there are changes in neurotrophins or corticotrophin releasing hormones associated with depression. New interventions to stop the stress hormone cascade before it gets rolling are being thought about; an input to psychopharmacology from the endocrinology.

\section{DISCUSSION}

An important reason for the high occurrence of refractory depression is our ignorance and tunnel vision about the aetiology of depression. With increasing knowledge of the physiological functions of monoamine neuronal system, the monoamine hypothesis is still in the forefront of scientific research. It is unquestionable that depression can stem from pure chemical causes and the present author does not question the value of the precious biological information gathered over the years but dubious about an extreme biological view held by some medical scientists.

Depression comprises a neurobiological or brain illness, but "medicalisation" of depression runs the risk of overlooking the psycho-social factors. Because of wide choice of antidepressants, clinicians are now able to tailor medication to symptomatology and patient's life situations and minimise side effects with selective prescribing. The invisibility of depression and, both subtle and flagrant discrimination against people with chronic depression make the treatment and management of this condition more complex. The newer techniques of brain imaging techniques like functional MRI or fMRI could unravel why some people may not be responding to the drug treatment.

Augmentation strategies have caught the maximum attention of the investigators, but robust evidence is lacking regarding its effectiveness. Lithium and thyroid augmentation are the two older established ones. Newer combination and augmentation strategies are only supported by case reports and open trial data; theoretical advantages of multiple mechanism are the basis of these approaches. This is done a la carte and sometimes in an irrational way. Much attention has not been paid to the evaluation of psychological approach. There is also a critical gap in data on the comparative benefits of 
various psychosocial therapies and herbal treatment with newer pharmacotherapies; gaps in knowledge should be filled including transcultural aspects. TMS is being experimented in several UK centres. VNS remains as a distant therapeutic promise.

Individual temperaments and their specific neurobiological substrates are still poorly understood, and this causes problems for the clinicians to find matching of antidepressants to specific temperament types. Practitioners have to depend on their own clinical judgement in deciding upon treatment and considering the diversity of clinical presentation, treatment intervention should be tailored to each clinical circumstance (Nelson 1998). What constitutes an adequate dose is not at all a wellresearched issue and it is the quality more important than the quantity of the therapy (US DHSS 1993).

Cognitive therapists sometimes run the risk of overlooking the biological correlates of depression. Beck has rightly stated that depression is due to a cognitive revolution following a coup in the mind and the coup takes place in the inaccessible dark valleys of the unconscious mind, resulting in the formation of the negative hypnotic script (Pandarakalam 2005). But cognitive theories do not fully explain the mechanism of how the unconscious mind prepares the "negative hypnotic script". A sequential strategy encompassing pharmacotherapy in the acute phase of illness and CBT in the residual phase has been found to be effective in decreasing relapse rate of TRD.

\section{Acknowledgements: None.}

\section{Conflict of interest: None to declare.}

\section{References}

1. American Psychiatric Association: Diagnostic and Statistical Manual of Mental Disorder ( $5^{\text {th }}$ edition) (DSM5) $A P A, 2013$

2. Anderson M Ian: We all know what we mean by treatmentresistant depression-don't we? Br J Psychiatry 2018, 212:259-261

3. Anderson M Ian: Drug treatment of depression: reflections on the evidence. Advances in Psychiatric Treatment 2003; 9:11-20

4. Bajbouj M, Merkl A, Schiaepfer TE: Two-year outcome of vagus nerve stimulation in treatment-resistant depression. J. Clin. Psychopharmacol 2010; 30:273-281

5. Barbee JG, Thompson TR, Jamhour NJ: A double-blind placebo-controlled trial of lamotrigine as an antidepressant augmentation agent in treatment-refractory unipolar depression. J. Clin Psychiatry 2011; 72:1405-1412

6. Bauer M, Dopfmer S: Lithium augmentation in treatment resistant depression; meta-analysis of placebo-controlled studies. J. Clin Psychopharmacol 1999; 19:427-434

7. Bauer M, Goetz T, Glenn T, Whybrow PC: The thyroid brain interaction in thyroid disorders and mood disorders Neuroendocrinology 2008; 20:1101-1114
8. Bauer M, Adli M, Bshcor T, Whybrow T: Lithium's emerging role in the treatment of refractory major depressive episodes: augmentation of antidepressants. Neuro-psychobiology 2010; 62:36-44

9. Bobo WV, Voort R, Croarkin PE, Leung JG, Tye SJ, Frye MA: Ketamine for treatment resistant unipolar and bipolar major depression: critical review and implications for clinical practice. Depress Anxiety 2016; 33:698-710

10. Bhugra Dinesh: Foreword. In Treatment Resistant Depression. Siegfried Kasper \& Stuart Montgomery. Wiley Black-Well, London, 2013

11. Cleare A, Pariante CM, Young AH, Anderson IM, Christmas D, Cowen PJ: Evidence based guidelines for treating depressive disorders with antidepressants: a revision of the 2008 for British Psychopharmacology association guidelines. J Psychopharmacol 2015; 29:459-25

12. Cooper-Kazaz R, Apter JT, Cohen R: Combined treatment with sertraline and liothyronine in major depression: a randomized, double blinded, placebo-controlled trial. Arch. Gene. Psychiatry 2007; 64:679-688

13. Crown WH, Finkelstein S, Berndt ER: The impact of treatment resistance in health care utilisation and costs. J. Clin Psychiatry 2002; 63:963-971

14. Davidson JRT, Gadde KM, Fairbank JA: Effect of Hypercium pereforatumin major depressive disorder: a randomised controlled trial. JAMA 2002; 287:1807-1814

15. Depression Guideline Panel: Depression in primary care, Vol 2 Treatment of major depression. Clinical practice guideline, No 5. pp71-86. Department of Health and Human Services. Rockville, MD, 1993

16. Devarajan S, Dursun SM: Citalopram plus reboxetine in treatment-resistant depression. Can J Psychiatry 2000; 45:489-490

17. Driessen E, Cuijpers $P$, de Maat $S$. C: The efficacy of short term psychodynamic psychotherapy for depression: a metanalysis. Clin Psychol. Rev 2010; 30 91:25-36

18. Dursun SM and Devarajan S: Reboxetine plus citalopram for refractory depression not responding to venlafaxine; possible mechanisms. Psychopharmacology 2001; 153:497-498

19. US DHSS: Depression in primary care: treatment guidelines. Rockville: AHCPR, 1993

20. Fava M: Augmentation and combination strategies in treatment resistant depression. J Clin Psychiatry 2001; 62(Suppl 80):4-11

21. Fink M: Electroconvulsive therapy in medication-resistant depression. In Amsterdam JD, Horning M, Nierenberg AA. eds. Treatment resistant Mood Disorders. Cambridge University Press, United Kingdom 223-238, 2001

22. Fornaro M, Giosue P: Current nosology of treatment resistant depression: a controversy of resistant depression. Clinical Practice, Epidemiology, Mental Health 2010; 4:20-24

23. Freedman SJ, Favu M, Kienke AS, White CN, Nierenbery AA, Rosenhan, JF: Partial response, non-response and relapse on SSRIs in major depression; A current "next step "practice. J clin psychiatry 2000; 61:403-407

24. Forbes $N$ and Rogers T: Combination therapy for treatment resistant depression. Progress in Neurology and Psychiatry 2003; 7:10-14

25. Gaynes BN, Lux LJ, Lloyd SW: Non-pharmacological interventions for treatment resistant depression in adults. Agency for Healthcare Research and Quality. Rockville. MD, USA, 2011 
26. Greenberg PE, Fournier AA, Sisitsky T, Pike CT, Kessler $R C$ : The economic burden of adults with major depressive disorder in the United States (2005 and 2010). J Clin Psychiatry 2015; 76:155-62. doi: 10.4088/JCP.14m09298

27. Harkin A, Kelly JP, McNamara M, Conner TJ, Dreg K, Leonard BE: Activity and onset of action of reboxetine and effect of combination with sertraline in an animal model of depression. Eur J. Pharmacology 1999; 364:123-32

28. Hollinghurst S, Carroll EF, Abei A: Cost-effectiveness of cognitive-behavioural therapy as an adjunct to pharmacotherapy for treatment resistant depression in primary care: economic evaluation of the CoBalT Trial. Br J Psychiatry 2014; 204:69-76

29. Iversen Leslie: Neurotransmitter transporters and their impact on the development of psychopharmacology. British Journal of Pharmacology 2003; 147:S82-S88. doi:10.1038/sj.bjp.0706428

30. Ivkovic M, Damjanovic A, Jovanovic A: Lamotrigine versus lithium augmentation of antidepressant therapy in treatment resistant depression: efficacy and tolerability. Psychiatiar Danub 2009; 21:187-193

31. Jacobsen FM: Possible augmentation of antidepressant response by buspirone. J Clin Psychiatry 1991; 52:217-220

32. Judd LL: Psychosocial disability during the long-term course of unipolar major depressive disorder. Arch Gen Psychiatry 2000; 57:375-380

33. Kasper $S \&$ Montgomery AS: Treatment-Resistant Depression. Wiley Blackwell, London, 2013

34. Kellner CH: The CORE/PRIDE Work Group. Right Unilateral Ultrabrief Pulse ECT in Geriatric Depression: Phase 1 of the PRIDE Study. Published online July 15, 2016 a. http://ajp.psychiatryonline.org/doi/10.1176/appi.ajp.2016. 15081101

35. Kellner CH: The CORE/PRIDE Work Group. A Novel Strategy for Continuation ECT in Geriatric Depression: Phase 2 of the PRIDE Study. Published online July 15, 2016b. http://dx.doi.org/10.1176/appi.ajp.2016.16010118

36. Kirsch I \& Lynn SJ: The altered state of hypnosis: Changes in the theoretical landscape. American Psychologist 1995; $50: 846-858$

37. Kirsch I: Hypnosis in Psychotherapy: Efficacy and mechanisms. Contemporary hypnosis 1996; 13:109-114

38. Kleenblatt J, Bletlzer F, Kilarski LL, Bschor T, Kohler S: Efficacy of off-label augmentation in unipolar depression: a systematic review of evidence. Eur Psychopharmacol 2017; 27:423-41

39. Maes M, Libbrecht I, Van Hunul F: Pindol and mianserin augmenting the antidepressant action of fluoxetine in hospitalized major depressed patients including those with treatment resistant. J Clin Psychopharmacol 1999; 19:177-182

40. Mihara K, Nakamura A, Nemeto K, Nagai G, Kagawa $S$, Suzuki $T$ et al.: Lamotrigine augmentation therapy in a case with treatment-resistant unipolar depression that showed insufficient response to electroconvulsive therapy. Psychiatry and clinical neurosciences 2016; 70:126

41. McAllister-Williams RH, Christmas DMB, Cleare AJ: Multiple therapy -resistant major depressive disorder: a clinically important concept. Br J Psychiatry 2018, 212:274-278

42. Montigny de C: Lithium addition to treatment resistant depression. Int. Clinical Psychiatry 1994; 9:31-35
43. Montgomery SA, Hubner WD, Grigoleic HG: Efficacy and tolerability of St John's wart extract compared to placebo in patients with mild to moderate depressive disorder. Phytomedicine 2000; 7(Suppl 11):7

44. Moller H-J, Seemuller F, Schennach R: Treatment Resistant Depression: A Separate Disorder- A new Approach, Chapter in Treatment- Resistant Depression by Kasper \& Montgomery. Wiley Blackwell, London, 2013

45. National institute for Clinical Excellence: Guidelines on the use of Electroconvulsive Therapy, London, NICE, 2003

46. Nelson JC: Combining drug treatment strategies for major depression. Psych Ann 1998; 28:197-202

47. O'Reardon JP, Solvason HB, Janicak PG: Efficacy and safety of transcranial magnetic stimulation in the acute treatment of major depression: a multisite randomised controlled trial; Biological Psychiatry 2007; 62:1208-1216

48. Ostroff RB, Nelson JC: Risperidone augmentation of SSRI in major depression. J Clin Psychopharmacol 1999; 60:256-259

49. Pae CU, Lim HK, Han C, Neena A, Lee C, Paktor AA: Selegiline transdermal system: current awareness and promise. Progress Neuropsychopharmacol Bio Psychiatry 2007; 31:1153-63

50. Pandarakalam JP: Refractory Depression. Modern Medicine 2004; 34:32-39

51. Pandarakalam James Paul: Are the hypnotherapeutic views of depression valuable? Modern Medicine 2005; 35:21-26

52. Pandarakalam JP: Bio-cognitive and quantum views of depression. American Journal of Psychiatry and Neurosciences 2018; 6:33-45. doi: 16.11648/j.ajpn.20180602.12

53. Parti Rajiv and Perry Paul: Dying to Wake up. Atria Books, New York, 2016

54. Perils R: The role of Pharmacogenetic $s$ in TRD progress and abstracts of the American Psychiatric Association $161^{\text {st }}$ annual meeting, May 3-8, Washington DC Symposium No10D, 2008

55. Posternack MA and Zimmerman: Switching versus augmentation: a prospective naturalistic comparison in depression, treatment resistant patients. $J$ Clinic Psychiatry 2001; 62:135-142

56. Power MJ: Cognitive therapy an outline of theory, practice and problems. Br J Psychotherapy 1989; 5:544554950

57. Reid PM, Shajahan PM, Glabus MF, Ebmeir KP: Transcranial magnetic stimulation in depression. $\mathrm{Br} J$ Psychiatry 1998; 173:449-452

58. Ribeiro DJ, Huang X, Fox RK, Franklin CJ: Depression and hopelessness as risk factors for suicide ideation, attempts and death: meta-analysis of longitudinal studies. The Br J Psychiatry 2018; 212:279-286

59. Rosenbaum Jerrod, Henninger George: Vagus Nerve Stimulation for Treatment Resistant Depression. Biological Psychiatry 2000; 47:273

60. Serrati A, Olgiati P, Bajo E: A model to incorporate genetic testing (5-HTTLPR) in pharmacological treatment of major depressive disorders. World J Bio Psychiatry 2011; 12:501-515

61. Shelton RC: Treatment options of Refractory depression. J Clin Psychiatry 1999; 60(Suppl 4):57-61

62. Shelton RC, Keller MB, Galenberg AJ: Effectiveness of St John's wart in major depression. JAMMA 2001; 285:1978-1986 
63. Shelton RC, Tollefson GG, Tohen M: A novel augmentation strategy for treatment resistant depression. Eur Psychiatry 2007; 17(Suppl 1):98

64. Sussman N: Neurochemistry of serotonin and depression. Primary Psychiatry 1995; 3:28-33

65. Sugawara Hiroko, Sakamoto Kaoru, Harada Tsuyoto, Ishigooka Jun: Predictors of efficacy in lithium augmentation for treatment resistant depression. Journal of Affective disorders 2010; 125:165-168

66. Souery D: Treatment resistant depression: methodological overview and operational criteria. Eur Psychopharmacol 1999; 9:83-91

67. Thase ME, Rush AJ: When at first you don't succeed: sequential strategies for antidepressant non-responders. J. Clin Psychiatry 1997; 58(Suppl 13):23-29

68. Thomas M Christine and Morris Stephen: Cost of depression in adults in England in 2000. Br J Psychiatry 2003; 183:514-519
69. Trivedi $M H$, Kleiber BA: Algorithm for the treatment of chronic depression. J Clin Psychiatry 2001; 62(Suppl 6):20-29

70. Trivedi RB, Nieuwsama JA, Williams JW Jr, Baker D: Evidence synthesis for determining the efficacy of psychotherapy for treatment resistant depression, Department of Veterans Affairs. Washington DC, USA, 2009

71. Vera De Carlo, Calati Raffaella, Seretti Alessandro: Socio-demographic and clinical predictors of nonresponse/non-remission in treatment resistant depressed patients: A systematic review. Psychiatry Research 2016; 249:421-430

72. Wiles N, Thomas L, Abel A, Barnes Carroll F, Ridgway N: Clinical and Cost effectiveness of cognitive behavioural therapy as an adjunct to pharmacotherapy for treatment resistant depression in primary care: The CoBalT randomised controlled trial. Health Techno Assess 2014; 18:1-16

Correspondence:

James Paul Pandarakalam, MBBS, DPM, RCPSI, DCP, MDCH

Department of Psychiatry, Northwest Boroughs Healthcare NHS Foundation Trust, Hollins park Hospital

Warrington WA2 8WN, United Kingdom

E-mail: James.pandarakalam@nwbh.nhs.uk; jpandarak@hotmail.co.uk 